\title{
REPORTE DE ECTOPARÁSITOS EN AVES DE CAUTIVERIO, Y SU CONTROL
}

\author{
Report of ectoparasites in captive birds and their control
}

\author{
Nayeli Joceline De La Crúz-Romero ${ }^{1}$, Vanessa Lizeth Gudiño-Mendoza ${ }^{1}$, Carlos \\ Manuel Ocegueda-Gutiérrez ${ }^{1}$, Ana Belén Solorzano-Mazariegos ${ }^{1}$, Elizabeth Abigail \\ Trejo-Moya ${ }^{1}$ y José Ricardo Cuéllar-Pérez ${ }^{2 *}$
}

\begin{abstract}
${ }^{1}$ Estudiante de la Licenciatura de Medicina Veterinaria y Zootecnia, CUCBA, Universidad de Guadalajara.

${ }^{2}$ Laboratorio de Morfofisiología, Departamento de Medicina Veterinaria. CUCBA, Universidad de Guadalajara.
\end{abstract}

*Autor para correspondencia: ricardo.cuellarp@academicos.udg.mx

\section{Resumen}

México se destaca a nivel mundial entre los primeros diez países exportadores de aves silvestres; el interés de poseer un ave para muchos mexicanos se incrementa, sin embargo, no se tiene el conocimiento necesario por parte de la población, para mantenerlos en buenas condiciones. En México existen cerca del 10\% de especies de aves del mundo, con un aproximado de 1,060 especies. Extraerlas de su hábitat natural es una práctica que se realiza en el país, por parte del comercio no regulado. Debido al desconocimiento y a la falta de responsabilidad de la población que gusta de tener aves en cautiverio, no se procura el bienestar de estos animales en ambientes adecuados y esto provoca la presencia de algunas especies de parásitos artrópodos externos, llamados ectoparásitos. Los ectoparásitos pueden causar enfermedades en humanos, animales domésticos y silvestres, actuando como reservorio de importantes patógenos que pueden transmitir infecciones zoonóticas. En este trabajo, se revisaron casos clínicos de aves domésticas con presencia de ectoparásitos, obtenidos de Médicos Veterinarios de la Zona Metropolitana de Guadalajara. En todos los casos analizados, se demostró la presencia del ectoparásito Knemidokoptes spp. causante de la sarna; el tratamiento más utilizado para eliminar la parasitosis es la ivermectina tópica, y el principal factor de riesgo identificado es la falta de limpieza de los alojamientos de estas aves. Un tratamiento oportuno para estas infecciones parasitarias es capaz de prevenir la muerte de estas aves, además, cuidar la alimentación y llevar a cabo un método profiláctico necesario para cada especie mejorará la calidad de vida de estas aves en cautiverio.

Palabras clave: Aves, ectoparásitos, Ivermectina, Melopsittacus undulatus, Knemidocoptes.
Abstract
Mexico is found in the top 10 countries worldwide that export wild birds. While there is an increasing demand for owning birds among Mexicans, there is insufficient information on how to appropriately care for them. Mexico is home to approximately 1,060 of the world's bird species, roughly $10 \%$. Removal of these birds from their natural habitat for commercial purposes without regulation is a practice widely undertaken in the country. As a result of 
the lack of knowledge and irresponsible practices while keeping these birds in captivity, their well-being is not maintained. They therefore become susceptible to the proliferation of external arthropod parasites, called ectoparasites. Ectoparasites can cause disease in humans, domestic and wild animals by acting as a reservoir for important pathogens that can transmit zoonotic infections. In this work, clinical cases of domestic birds obtained from Veterinarians of the Metropolitan Area of Guadalajara that were infected by ectoparasites were reviewed. In all the cases analyzed, the presence of the ectoparasite Knemidokoptes spp., cause

\section{Introducción}

En México existen alrededor de 1,060 especies de aves, que representa cerca de $10 \%$ de especies de todo el mundo y es reconocido entre los diez primeros exportadores de aves silvestre (ÍñigoElias et al., 1997). Tradicionalmente las aves han tenido una importancia cultural relevante, ya que muchas especies han sido utilizadas como alimento o se han vinculado en procesos simbólicos y medicinales. Una de las prácticas más arraigadas es poseer un ave, ya sea para ornamentar el entorno o gozar de sus cánticos y compañía siendo extraídas de su medio natural (Gomez et al., 2005b). Debido a la demanda que poseen estas aves, se ha propiciado el comercio formal e informal, que en las últimas décadas ha incrementado de manera no regulada (Gobierno de México, 2019; Navarro-Sigüenza et al., 2014). El proceso de extracción y el mantenimiento de las aves en los hogares puede comprometer la salud de éstas y reducir su esperanza de vida, se han comercializado de tal grado que actualmente tienen problemas de supervivencia, y desarrollan enfermedades en humanos, animales domésticos y silvestres, actuando como of scabies, was found. The most commonly used treatment for elimination of parasitosis is topical Ivermectin, and the main risk factor identified is the lack of cleanliness in bird housing. A timely treatment for these parasitic infections is able to prevent the death of these birds. Additionally, a proper diet and carrying out a necessary prophylactic method for each species will improve their quality of life in captivity

Key words: Birds, ectoparasites, Ivermectin, Melopsittacus undulatus, Knemidocoptes.

vectores biológicos y mecánicos de importantes patógenos (Parra et al., 2010). Una de las especies más comercializadas en nuestro país son los Psitácidos, y de las 21 especies que habitan en México, 15 están protegidas por la legislación mexicana. Las condiciones de vida en cautiverio deben de ser apropiadas para cada especie de aves y debe contribuir tanto a su salud como a su comodidad. Gómez et al. en el 2005a, publicó un artículo donde señala las principales condiciones que deben tomarse en cuenta para el adecuado cautiverio de aves de ornato y compañía, y las especies que se encuentran en peligro de extinción. Las principales enfermedades que atacan a estas aves en cautiverio son ocasionadas por parásitos que. Muchas especies de aves albergan una fauna parasitaria diversa; aunque algunas especies son específicas del huésped y pasan todo su ciclo de vida en él (Saavedra et al., 2014). En las ciencias médicas y veterinarias, generalmente, se acepta como "parásito" solamente a eucariotas y metazoarios; es decir, protozoarios, helmintos y artrópodos (Benavides, 2012). Los artrópodos son animales invertebrados que incluyen una gran variedad de especies, clases y órdenes, 
ya que tiene una gran capacidad de adaptación se encuentra prácticamente en todos los rincones del planeta (Pinkus, 2010). Las características comunes de los artrópodos son poseer un exoesqueleto, un polisacárido que recubre su cuerpo y les proporciona protección física además de resistencia a la desecación, y patas articuladas (Fernandez et al., 2008). Dentro de este phylum la mayoría son ectoparásitos, los cuales representan un grupo sumamente heterogéneo en cuanto a su taxonomía y biología, que incluye a los ácaros, garrapatas, pulgas y piojos (Lareschi, 2017). Estos organismos son importantes en la medicina veterinaria debido a que son capaces de causar lesiones cutáneas, inducir una respuesta inmunopatológica y transmitir agentes patógenos ya que pueden ser zoonóticos o transmitir infecciones zoonóticas.

\section{Artrópodos que afectan con frecuencia la piel}

Las estrategias de control en el manejo de los ectoparásitos deben tener en cuenta ciertos factores como, tipo de parásito, características del animal (especie, edad, tipo de pelo), disponibilidad de productos farmacéuticos, vías de aplicaciones y duración del tratamiento. A continuación, se presentan algunos de los parásitos externos más frecuentes y los problemas que pueden producir.

La sarna (Knemidokoptes spp.) se puede desarrollar en forma completa en el ave, por lo que su transmisión es directa o por contacto estrecho. Los signos clínicos que la diferencian son la inducción de proliferación de la epidermis de la piel sin plumas (patas, cera, alrededor del pico, ojos y ventus), con aspecto de panal de abejas y se ve más frecuente en periquitos australianos y canarios. En otras aves, la sarna suele causar pérdida de plumas, prurito y piel descamada, sobre todo en cabeza y cuello, en su aspecto macroscópico se puede visualizar lesiones en pico y patas. Para la confirmación de sarna de haber una observación directa de raspajes o biopsias de piel y la respuesta clínica al tratamiento (Bruno y Albuquerque, 2008; Dabert et al., 2012). Los ácaros rojos (Dermanyssus spp., Ornithonyssus spp., etc.) son hematófagos y es más común que se alimenten por la noche, a diferencia de Ornithonyssus spp., que siempre está sobre el ave. La transmisión de este ácaro es por contacto directo y es muy común en paseriformes, las picaduras de ácaros en animales conducen a un comportamiento de picoteo que les causa heridas graves y aumenta la probabilidad de canibalismo, incluso llegando a generar agresividad e irritación. Sus signos clínicos se comprenden por la falta de descanso, irritabilidad, debilidad, anemia, prurito, falta de desarrollo. Se puede diagnosticar haciendo una identificación del parásito en el ave o en la jaula, preferentemente de noche (Sparagano et al., 2016). Los ácaros de plumas y cañón (Epidermoptes spp., Dermatophytes spp., Syringophilus spp., etc.) se realizan en forma completa sobre el ave y se transmiten por contacto directo. Estos no son hematófagos, es común en paseriformes, columbiformes y galliformes $y$ normalmente presenta en el individuo la falta de descanso, irritabilidad, prurito, plumas roídas o agujereadas, pero cuando la infestación es intensa llega a ocasionar reacciones alérgicas $\mathrm{o}$ favorecer la contaminación por hongos y bacterias. Para el diagnóstico de estos ácaros es bueno que se haga una identificación del parásito (adultos, ninfas, larvas, huevos) en las plumas (Santa Cruz et al., 2008). Las garrapatas (Argas spp., Ixodes spp., Amblyomma spp., Ricinus spp., Rhipicephalus spp., etc.), son hematófagas, pero poco patógenas y se alimentan 
preferentemente de noche afectando a la gran mayoría de las especies las cuales causan ansiedad, debilidad y anemia. Se encuentran en forma completa o incompleta sobre el ave, transmitiendo por contacto directo. Para obtener un buen diagnóstico tiene que haber una identificación del parásito en el ave (Goodman et al., 2005; GuzmánCornejo y Robbins, 2010). Insectos: piojos masticadores (Mallophaga spp.), pulgas (Ceratophyllus spp., Dasypsyllus spp., Hectopsilla spp.) son conocidas por transmitir enfermedades (LujánVega y Gómez-Puerta, 2016), también están las moscas hippoboscidae (Pseudolincha spp., Dermatobia spp.), mosquitos (Culex spp., Aedes spp., Anopheles spp.) y chinches. Estos se realizan en forma completa $o$ incompleta sobre el ave transmitiendose por contacto directo; sólo algunos son hematófagos (pulgas, chinches, moscas chupadoras, mosquitos), otros se alimentan con las barbas y escamas de las plumas (piojos masticadores) o de tejidos (miasis) y son poco patógenos afectando aun así a todas las especies de aves. Los signos clínicos que se presentan son la falta de descanso, irritabilidad, prurito, plumas roídas o agujereadas, picaduras, gusanos en el subcutáneo, debilidad, anemia y para el diagnóstico es bueno la identificación del parásito (adultos, ninfas, larvas, huevos) en las plumas o en el ave (Rodríguez-Ortega et al., 2018).

El Cuadro 1, muestra en resumen los principales ectoparásitos que afectan a las aves, los signos clínicos que se pueden presentar posterior a la infección, diagnósticos $\mathrm{y}$, el tratamiento como prevención y erradicación.

Las aves de ornato son muy susceptibles al ataque de ectoparásitos originando una reacción de estrés que incrementa la tasa de corticosteroides, reduciendo el consumo de alimentos, baja de respuesta inmune y aumenta la susceptibilidad a enfermedades. El confinamiento, puede favorecer la multiplicación y dispersión de los ectoparásitos en los galpones de producción y el aumento de efectos negativos del parasitismo en aves (Brown, 1972). Por lo tanto, la identificación de ectoparásitos es trascendental en el campo de la Medicina Veterinaria, debido a que es un primer paso para evitar que estos afecten severamente a las especies animales, en este caso, las aves de cautiverio; ya sea de modo directo o indirecto; al llegar a actuar como vectores biológicos y mecánicos de diversos agentes patógenos (SaavedraOrjuela et al., 2014). La Ivermectina es un agente antihelmíntico semisintético. Es una avermectina con un grupo de lactona pentacíclica de dieciséis miembros (es decir, un disacárido de lactona macrocíclica) derivada de la bacteria del suelo Streptomyces avermitilis. La Ivermectina se une selectivamente y con alta afinidad a los canales iónicos de cloruro activados por glutamato en las células nerviosas y musculares de invertebrados de las microfilarias. Esta unión provoca un aumento de la permeabilidad de la membrana celular a los iones de cloruro y da como resultado la hiperpolarización de la célula, lo que conduce a la parálisis y la muerte del parásito (Yates y Wolstenholme, 2004). El objetivo de este trabajo fue reportar los factores que facilitan el diagnóstico de la presencia de ectoparásitos en aves de cautiverio, para difundir conocimientos de la función de los ectoparásitos más comunes en aves de cautiverio, al igual que las medidas de prevención y control necesarias para evitar que estos parásitos se propaguen entre la producción avícola, dañando su bienestar y la economía de los productores, cuando de aves de corral se trata. Además, cabe mencionar que las aves forman parte importante en las 
sociedades humanas, ya que poseen

espacio y cultura.

usos y valores que varían con el tiempo,

Cuadro 1.- Grupos principales de parásitos externos o ectoparásitos, que afectan a las aves.

\begin{tabular}{|c|c|c|c|}
\hline Tipo de parásito & Signos clínicos & $\begin{array}{c}\text { Diagnóstico, } \\
\text { diagnóstico } \\
\text { diferencial (DD) y } \\
\text { cambios post } \\
\text { mortem (PM) }\end{array}$ & $\begin{array}{c}\text { Tratamiento o } \\
\text { prevención }\end{array}$ \\
\hline $\begin{array}{l}\text { Chinches } \\
\text { (Hemiptera Cimicidae) }\end{array}$ & $\begin{array}{l}\text { Mal aspecto, irritabilidad, } \\
\text { intranquilidad } \\
\text { (especialmente por la } \\
\text { noche), inflamación e } \\
\text { irritación de la dermis }\end{array}$ & $\begin{array}{l}\text { Descubrimiento de } \\
\text { parásitos en las aves } \\
\text { (especialmente por la } \\
\text { noche) DD: otros parásitos } \\
\text { hematófagos nocturnos }\end{array}$ & $\begin{array}{l}\text { Desinfección del entorno, } \\
\text { tratamiento de las aves con } \\
\text { polvo de piretrina }\end{array}$ \\
\hline $\begin{array}{l}\text { Piojos masticadores } \\
\text { (Phthiraptera-Mallophaga) }\end{array}$ & $\begin{array}{l}\text { Rascado, picor, plumaje } \\
\text { opaco y sin brillo, } \\
\text { plumas enredadas y } \\
\text { dañadas }\end{array}$ & $\begin{array}{l}\text { Presencia de huevosunidos } \\
\text { a las plumas y de ninfas y } \\
\text { parásitos adultos en las } \\
\text { plumas PM: plumas } \\
\text { dañadas, sin brillo, } \\
\text { presencia de huevos y de } \\
\text { adultos muertos en el } \\
\text { plumaje }\end{array}$ & $\begin{array}{l}\text { Insecticidas con polvos de } \\
\text { piretrina, rociador de } \\
\text { rotenona. Tratamiento } 1 \text { vez a } \\
\text { la semana, 2-3 veces, } \\
\text { utilizando también la técnica } \\
\text { de la bolsa de plástico }\end{array}$ \\
\hline $\begin{array}{l}\text { Dípteros (Diptera): mosquitos } \\
\text { (Culicidae); mosquitos } \\
\text { picadores (Ceratopogonidae); } \\
\text { moscas negras (Simuliidae); } \\
\text { moscardas (Calliphoridae); } \\
\text { moscas hipobocidos } \\
\text { (Hippoboscidae) }\end{array}$ & $\begin{array}{l}\text { Anemia, irritación y, } \\
\text { dermatitis de las partes } \\
\text { del organismo no } \\
\text { cubiertas por plumas, } \\
\text { presencia de huevos o } \\
\text { larvas alrededor de los } \\
\text { orificios naturales o de } \\
\text { las heridas de la piel }\end{array}$ & $\begin{array}{l}\text { Presencia de insectos } \\
\text { picadores en las jaulas o } \\
\text { instalaciones, o en las aves } \\
\text { PM: inflamación de la piel, } \\
\text { pérdida de peso, presencia } \\
\text { en algunos casos de huevos } \\
\text { y/o larvas; inmediatamente } \\
\text { después de la muerte, los } \\
\text { parásitos abandonan el } \\
\text { cuerpo }\end{array}$ & $\begin{array}{l}\text { Mosquitos: mosquiteras, } \\
\text { piretrinas } \\
\text { Otros insectos voladores: es } \\
\text { muy difícil erradicarlos. } \\
\text { Moscas: eliminar las larvas de } \\
\text { las heridas a mano, } \\
\text { desinfectar con agua caliente } \\
\text { que contenga un desinfectante }\end{array}$ \\
\hline Pulgas (Siphonaptera) & $\begin{array}{l}\text { Irritación cutánea, } \\
\text { acicalamiento y } \\
\text { encrespamiento de las } \\
\text { plumas, pérdida de } \\
\text { sangre a través de la piel, } \\
\text { plumaje sucio y } \\
\text { apelmazado }\end{array}$ & $\begin{array}{l}\text { Identificación de los } \\
\text { huevos y las larvas, que } \\
\text { pueden encontrarse } \\
\text { principalmente en los nidos } \\
\text { y las crías Echidnophaga se } \\
\text { une por sí mismo a la } \\
\text { cabeza de las aves }\end{array}$ & $\begin{array}{l}\text { Desinfección del entorno, } \\
\text { tratamiento de las aves con } \\
\text { polvo de piretrina }\end{array}$ \\
\hline Garrapatas (Ixodida) & $\begin{array}{l}\text { Anemia, irritación, picor } \\
\text { y lesiones de los tejidos, } \\
\text { falta de crecimiento, } \\
\text { muerte }\end{array}$ & $\begin{array}{l}\text { Descubrimiento de larvas, } \\
\text { ninfas o adultos en el } \\
\text { cuerpo de las aves }\end{array}$ & $\begin{array}{l}\text { Eliminación de los parásitos a } \\
\text { mano, tratamiento del entorno }\end{array}$ \\
\hline $\begin{array}{l}\text { Ácaros (Acari) Escarabajos } \\
\text { (Coleoptera) }\end{array}$ & $\begin{array}{l}\text { Irritación, picor y } \\
\text { lesiones de los tejidos }\end{array}$ & $\begin{array}{l}\text { Presencia de Dermestes sp. } \\
\text { u otras especies en los } \\
\text { nidos o las aves }\end{array}$ & $\begin{array}{l}\text { Eliminación de los parásitos a } \\
\text { mano, tratamiento del entorno }\end{array}$ \\
\hline
\end{tabular}

Tomado de: Zucca et al., 2010

\section{Materiales y métodos}

Este estudio se basó en la recolección de casos clínicos de aves infectadas con ectoparásitos y se realizó con ayuda de Médicos Veterinarios Zootecnistas egresados de la Universidad de Guadalajara y expertos en aves de la zona metropolitana. Se realizó una comparación y clasificación de los distintos agentes, entornos y tratamientos más comunes utilizados en el ambiente de las aves muestreadas. Se reportan las medidas de prevención y control más eficaces. Se recopilaron 10 casos clínicos dentro de la Zona Metropolitana de Guadalajara, que incluyeron las especies periquito autraliano (Melopsittacus undulatus), 
perico frente naranja (Eupsittula canicularis), canario silvestre (Serinus canaria), el pavorreal (Pavo cristatus), y el guacamayo (Ara militaris).

\section{Resultados y discusión}

De acuerdo a los datos obtenidos, la especie más afectada fue el periquito autraliano (Melopsittacus undulatus), seguida del perico frente naranja (Eupsittula canicularis) junto con el canario silvestre (Serinus canaria), el pavorreal (Pavo cristatus), y el guacamayo (Ara militaris). Los casos de periquitos australianos que se encontraron infectados con el ácaro
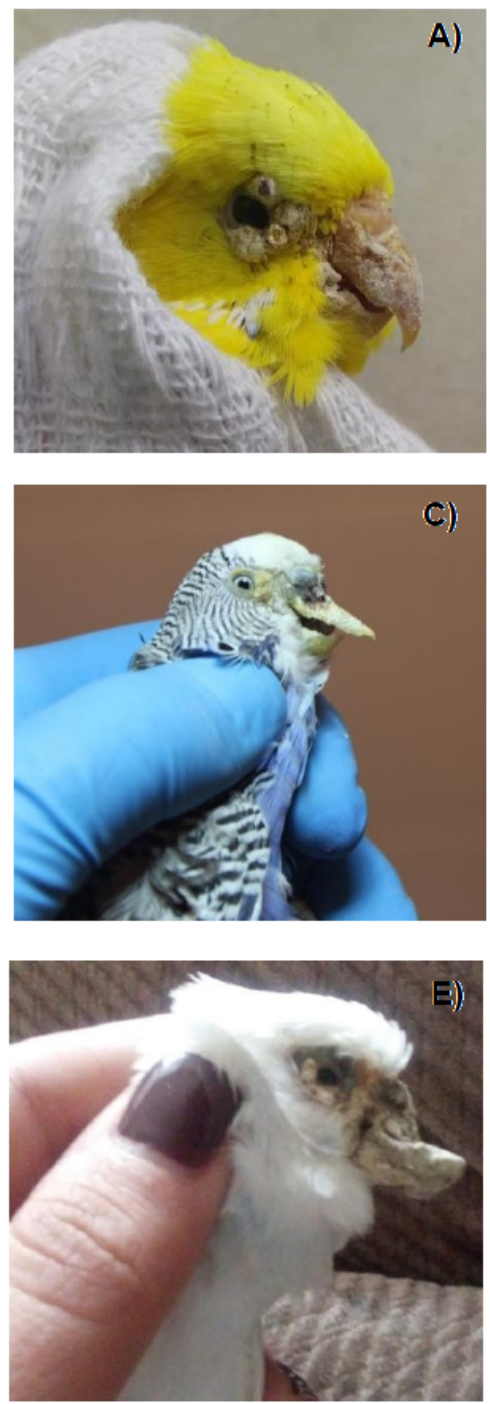

Knemidocoptes (Figura 1), presentaban deformidad en el pico, prurito, hiperqueratosis y lesiones en forma de panal; estas aves mostraban signos de desnutrición, pero sus dueños mencionaron no haber desparasitado a sus aves. La mayoría de estos casos compartían jaula con otra ave de la misma especie, la pareja de uno de estos casos murió por la infección parasitaria. El tratamiento farmacológico que se siguió fue la aplicación de Ivermectina tópica, una vez por semana durante un mes, en algunos de los casos, los médicos veterinarios lo complementaron con vitamina $\mathrm{E}$ en ungüento.
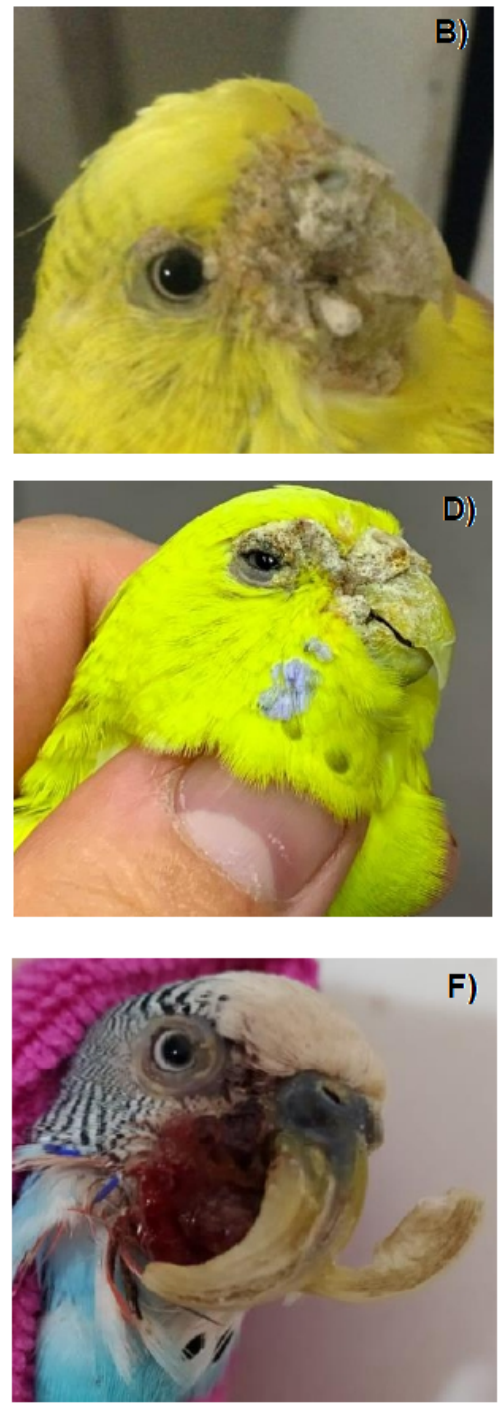

Figura 1. Casos clínicos de periquitos australianos (Melopsittacus undulatus), infestados con el ectoparásito Knemidokoptes spp. A) y B) proliferación del ácaro, C), E) y F) deformidad del pico e hiperqueratosis. 
En el caso del perico frente naranja, se menciona que fue infectada por pulgas y el dueño manifestó limpiar la jaula de forma cotidiana. Esta ave se encuentra en una jaula alejada de la luz solar y convive con otras aves de la misma especie, el dueño mencionó siempre mantener limpia la jaula. El tratamiento consistió en baños con agua de manzanilla, analgésico meloxicam y desparasitación con Ivermectina tópica (Figura 2). El canario estaba alojado en un aviario, y estaban en temporada de cría, el dueño hizo mención de que algunas crías morían durante los primeros cinco días de nacimiento. El canario revisado en este caso presentaba hematomas en todo el cuerpo y se diagnosticó que tenía infestación por ácaros, el tratamiento también se utilizó Ivermectina tópica para todas las aves del aviario y se hizo una fumigación de las jaulas y perchas. Para el caso del pavorreal, fueron tres aves de la misma especie provenientes de un rancho y con un estado de anemia, el tratamiento que se aplicó fue Ivermectina tópica (Figura $3)$.

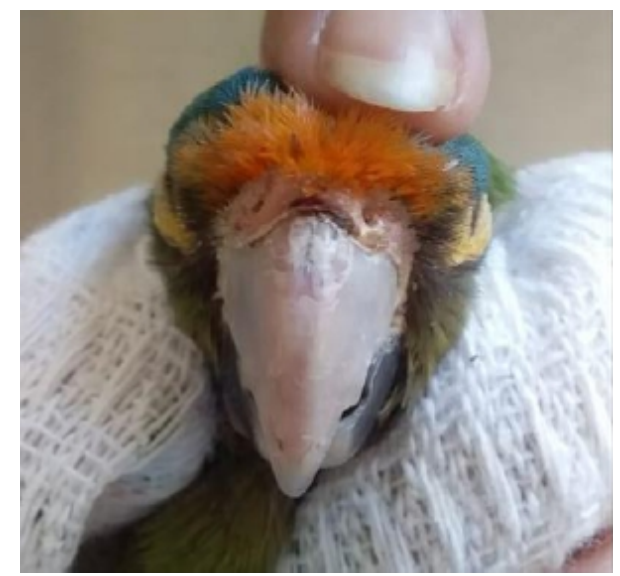

Fig. 2. Caso clínico del perico frente naranja (Eupsittula canicularis), infestado de pulgas.
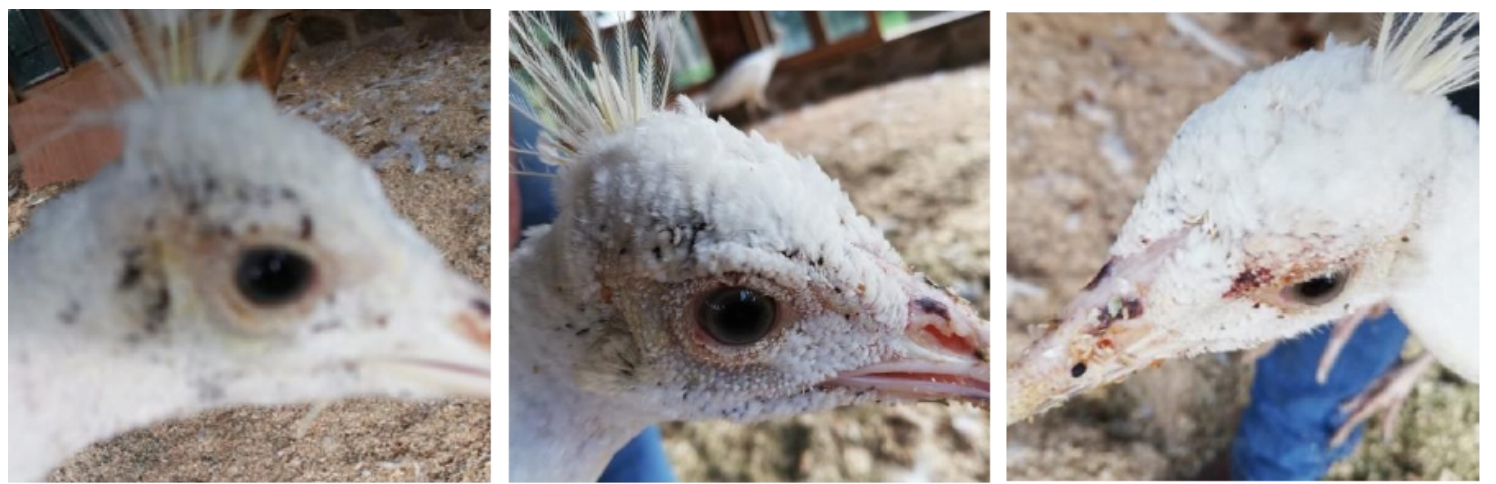

Figura 3. Caso clínico de tres pavorreales (Pavo cristatus), infestados con pulga de la especie Ceratophyllus spp.

Finalmente, en el caso del guacamayo se menciona que tenía infestación del ácaro Knemidocoptes aunque no pudo ser confirmado debido la gravedad con la que se presentó el ave, y de igual forma con los anteriores casos se aplicó Ivermectina tópica durante 4 meses (Figura 4). 


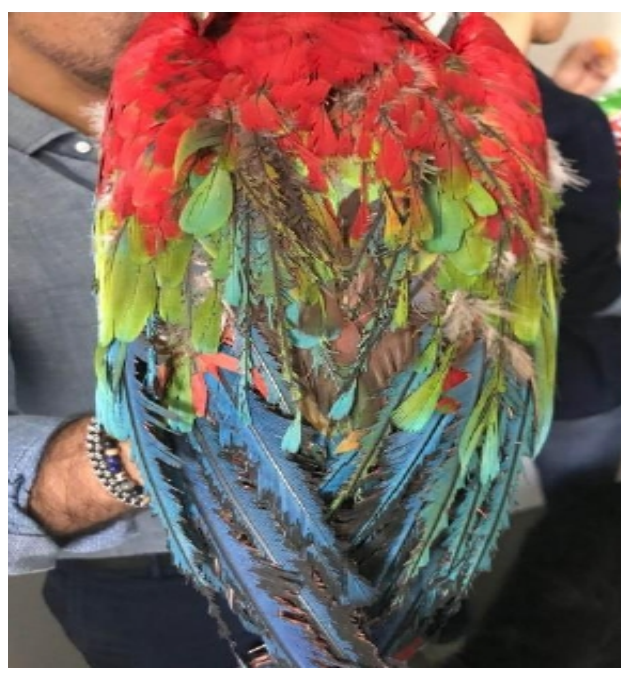

Figura 4. Caso clínico del guacamayo (Ara militaris), infestado con el ácaro Knemidocoptes.

De la gran cantidad de ectoparásitos existentes en aves de cautiverio; en forma descendente, el Knemidocoptes tiene un $80 \%$ de prevalencia; y con posible afinidad en la especie Melopsittacus undulatus, el Ceratophylus spp. tiene un $10 \%$ al igual que la pulga. Por lo que se considera que, dentro de la zona metropolitana de Guadalajara, el Knemidocoptes es más susceptible a infectar estas aves (Figura $5)$.

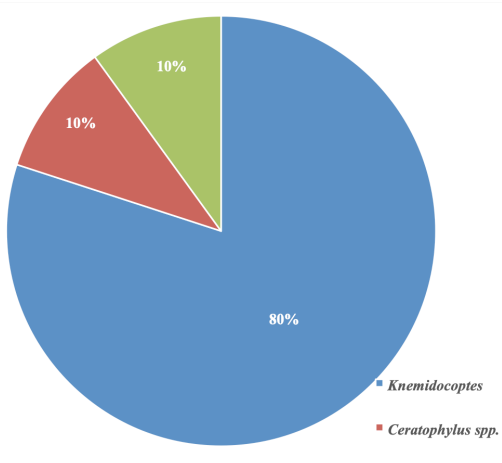

Figura 5. Proporción de ectoparásitos encontrados en los casos clínicos analizados

También, se determinó como tratamiento principal para el control de ectoparásitos en aves en cautiverio, la Ivermectina, ya que ésta funciona como antiparasitario de amplio espectro, con una variedad de dosis y métodos de aplicación en especies de aves (Figura 6).

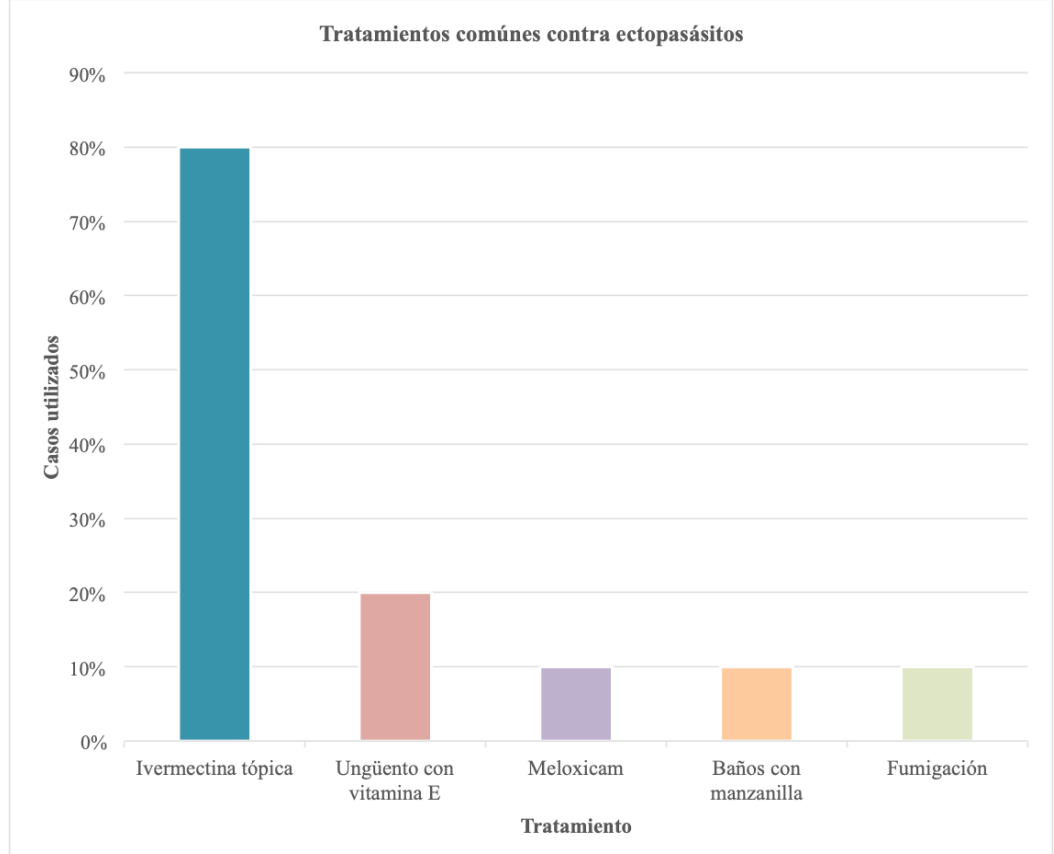

Fig. 6. Frecuencia de los tratamientos utilizados para controlar la infestación de ectoparásitos de los casos clínicos analizados. 
Múltiples reportes científicos demuestran que existe una eficacia confirmada del uso de Ivomec (Ivermectina) para el tratamiento de ectoparásitos de los pájaros ornamentales; no obstante, se especifica que, si este se da de forma incorrecta $y$ durante largos periodos de tiempo, se generarán efecto dañino como convulsiones, temblores, ceguera y hasta la muerte (Garcia et al., 2011; Klafke et al., 2012). En todos los casos clínicos analizados, el uso de esta Ivermectina fue de manera tópica; ya que la administración intramuscular de esta a las aves pequeñas puede ser tóxica, y de forma oral puede ser prácticamente difícil para el propietario, por lo que el líquido tópico "gota a gota" puede ser adecuada para el control de ectoparásitos. Kamal et al., (2012) demuestran que el uso repetido de la formulación líquida de Ivermectina no produce ninguna reacción adversa apreciable en las aves, ni se observa alguna reacción en las aves sometidas al ensayo de toxicidad.

El Ácaro Knemidocoptes es el ectoparásito que tiene más incidencia en los casos clínicos recopilados para este trabajo de investigación; sin embargo, estudios realizados en otros países, mencionan que los ectoparásitos con más prevalencia son los piojos, esto debido a que en sus resultados mencionan que la grulla corona, los gansos y el pavo real mostraron solo infestación de piojos, mientras la cigüeña marabú y el avestruz mostraron tanto piojos como infestaciones de garrapatas (Nijila et al., 2018). Por otro lado, en el estudio elaborado por Garcia et al. en 2018 se explica que los ectoparásitos con más repercusión son los ácaros plumícolas del género Pterophagus spp. y el ácaro de la superfamilia Pterolichoidea, ya que en sus resultados se observan las asociaciones de ectoparásitos y las aves con ambos ácaros se encontraban en mayor proporción, teniendo diferencias altamente significativas respecto al resto de las combinaciones parasitarias. Debido a la falta de continuidad o de término de los tratamientos con Ivermectina, los ectoparásitos comienzan a generar mecanismos de resistencia para este fármaco. De tal forma que, el empleo de organofosforados, carbamatos, piretroides y piretrinas mediante pulverización a alta presión ayuda en el tratamiento y control contra algunos de estos ectoparásitos en aves, sugiriendo, además, que una medida de control también puede ser la erradicación de nidos abandonados (Jofré et al., 2009). Finalmente, la limpieza a conciencia de todos los espacios en donde se encuentran las aves, así como un correcto seguimiento de calendarios de desparasitación acordado por el médico veterinario encargado son necesarios para reducir la prevalencia de ectoparásitos.

\section{Conclusión}

Los tratamientos que tienen un mayor impacto para combatir la infección por ectoparásitos son los organofosforados, carbamatos, piretroides e insecticidas resaltando con mayor uso en aves, la Ivermectina. El ácaro Knemidocoptes se encontró con mayor incidencia en este estudio con posibilidad de afinidad hacia la especie Melopsittacus undulatus, de igual manera es importante hacer énfasis en que la vía de administración del tratamiento antiparasitario depende de la naturaleza del fármaco siendo la Ivermectina recetada únicamente de manera tópica.

Las infestaciones por ectoparásitos provocan daños considerables en poblaciones aviares por lo que el uso correcto de los diferentes métodos de control es de suma importancia para las aves en cautiverio y su bienestar. En las 
aves en cautiverio, los mecanismos de búsqueda de los nutrientes necesarios pueden estar activos, sin embargo, no podrán ser desarrollarlos por hallarse confinadas, estas necesidades nutricionales deben ser cubiertas con dietas balanceadas aportadas por los dueños y de no ser así se afectará la salud de ellas, alternándose la normal manifestación de procesos fisiológicos, la reproducción de crecimiento de las crías, el cambio de plumas y la resistencia a las enfermedades. Confeccionar las raciones que satisfagan plenamente los requerimientos de las diversas especies de aves criadas en cautiverio aún hoy con los avances científicos existentes, continúa siendo un reto para nutricionistas, veterinarios y criadores, ya que cuando se habla de balance alimentario se hace conforme a elementos nutritivos del ave para que cubran sus necesidades y los excesos en diversos elementos muchas veces traen acumulaciones que pueden afectar también la salud de estas. Las aves en cautiverio suelen ser alojadas en jaulas, aviarios y corrales exteriores, las cuales deben ser limpiadas a fondo con desinfectante o un limpiador comercial para jaulas cada vez que se utiliza un ave nueva, a intervalos de tiempo determinados ya que se ensucian con

\section{Literatura citada}

Benavides, E. (2012). Enseñanza de la parasitología veterinaria a partir del uso de organismos vivos y tecnologías de la información y de la comunicación (TIC). Revista de Medicina Veterinaria, (23), 97-109. Retrieved January 18, 2021, from http://www.scielo.org.co/scielo.php ?script $=$ sci_arttext\&pid=S012293542012000100010\&lng=en\&tlng $=$ es. rapidez, al igual que por problemas con ácaros y otras plagas. La opción de jaula será más apropiada dependiendo de la especie, ya que el tamaño, la forma y el diseño del encierro debe de ser apropiado para la especie alojada y permitirle espacio sin hacinamiento para el movimiento natural del ave, todo esto como medidas profilácticas. Por último, se debe resaltar, como otra medida profiláctica indispensable, seguir las recomendaciones por parte de médicos veterinarios competentes para el manejo en cautiverio de las aves y motivar una cultura que promueva el llevar a consulta a las aves en cautiverio no solamente por motivos patológicos, sino rutinarios.
Brown, N. (1972.) The Effect of Host Beak Condition on the Size of Menacanthus Stramineus Populations ofDomestic Chickens. Poult Sci. 51(1):162-4. doi: 10.3382/ps.0510162.

Bruno, S., Albuquerque, D. (2008). Ocorrência e tratamento de sarna knemidocóptica (Knemidokoptes sp.) em aves de companhia atendidas na Faculdade de Veterinária da Universidade Federal Fluminense, RJ. Ciênc Rural 38: 1472-1475. doi: 10.1590/ S0103-84782008000500046

Dabert, J., Mihalca, A., Sándor, A. 
(2011). The first report of Knemidocoptes intermedius Fain et Macfarlane, $1967 \quad$ (Acari: Astigmata) in naturally infected European birds. Parasitol Res 109: 237- 240. doi: 10.1007/s00436011-2390-8

Fernández-Rubio, F., Moreno, L., Soriano, O. (2008). Artrópodos en medicina y veterinaria. $3^{\mathrm{a}}$ edición ampliada. Ministerio de Defensa. Madrid. p 643.

García, B., Hernández, D., Soler, F., Pérez-López, M. (2011). Empleo de ivermectina como parasiticida en ovino: posibles efectos tóxicos y repercusiones ambientales. AN. VET. (MURCIA) 27: 23-32. DOI: $10.6018 / \mathrm{j} / 160111$

Gobierno de México, (2019), La Relevancia de las aves en México, disponible en Internet en https://www.gob.mx/profepa/es/arti culos/la-relevancia-de-las-aves-enmexico2?idiom=es\#: :text $=$ Las $\% 2$ 0aves $\% 20$ son $\% 20$ parte $\% 20$ integral ,cetrer $\% \mathrm{C} 3 \% \mathrm{ADa} \% 2 \mathrm{C} \% 20$ arte $\% 20$ plumario $\% 20 \mathrm{y} \% 20$ recreaci $\% \mathrm{C} 3 \%$ B3n.

Gómez, G., Valadez, R., Teutli, C., Reyes, S.R. (2005a). Manejo en cautiverio de psitácidos utilizados como aves de ornato y compañía. AMMVEPE, 16(1): 5-17.

Gómez, G., Teutli, C., Reyes, S.R., Valadez, R. (2005b), Pájaros y otras aves utilizados como animales de ornato y compañía, AMMVEPE, 16(5): 129-139.

Goodman, J.L., Dennis, D.T. Sonenshine, D.E. (2005). Tickborne diseases of humans. American Society for Microbiology Press, Washington, $401 \mathrm{p}$.

Guzmán-Cornejo, C., Robbins, R.G. (2010). The genus Ixodes (Acari: Ixodidae) in Mexico: adult identification keys, diagnoses, hosts, and distribution. Revista Mexicana de Biodiversidad,
81:289-298

Íñigo-Elías E, Ramos MA. El comercio depsitácidos en México. En: Robinson JG Re-dford K. H (comp.) Uso y conservación dela vida silvestre neotropical, México: Fon-do de Cultura Económica; 1997.

Jofré, L., Noemí, I., Neira, P., Saavedra, T., Díaz, C. (2009). Acarosis y zoonosis relacionadas. Revista chilena de infectología, 26(3), 248257

Kamal, M.D., Sandersona, D., Nahara, K., Gestiera, A.W., Khanc, M.S., Hamid H. (2012). Dose titration, efficacy and safety of 'drop on' ivermectin for the management of knemidocoptes spp infestation in budgerigars. J App Pharm 03(04): 670-675.

Klafke, G.M., Castro-Janer, E., Mendes, M.C., Namindome, A., Schumaker, T.T.S. (2012). Applicability of in vitro bioassays for the diagnosis of ivermectin resistance in Rhipicephalus microplus (Acari: Ixodidae). Vet Parasitol, 184(24):212-20. doi: 10.1016/j.vetpar.2011.09.018. Epub 2011 Sep 17.

Lareschi, M. (2017). Artrópodos ectoparásitos. pp. 167-185. En: Drago, F.B. Macroparásitos. Diversidad y biología. Editorial de la Universidad Nacional de La Plata (EDULP). De la Plata, Uruguay. ISBN: 978-950-34-15214.

Luján-Vega, C., Gómez-Puerta, L.A. (2016). Contribución al estudio de pulgas (Insecta: Siphonaptera) en aves del Perú. Revista Peruana de Biología. Retrieved January 18, 2021, from https://www.researchgate.net/profile/C harlene_Lujan_Vega/publication/3052 80526_Contribucion_al_estudio_de_p ulgas_Insecta_Siphonaptera_en_aves_ del Peru/links/5786842e08ae36ad40a $695 \bar{b}$ 9.pdf 
Navarro-Sigüenza, A.G., RebónGallardo, M.F. Gordillo-Martínez, A., Peterson, A.T., BerlangaGarcía, H., Sánchez-González, L.A. (2014). Biodiversidad de aves en México. Revista Mexicana de Biodiversidad, Supl. 85: S476S495, DOI: $10.7550 /$ rmb. 41882

Nijila, H.L., Debi-Dore, J.D., Ombugadu, A., Dibal, M., Mafuyai, M.J. (2018). Survey of Ectoparasites Infesting Captive Birds in the Jos Museum Zoological Garden, North Central, Nigeria. Journal of Natural Sciences Research, 8(7); 36-40.

Parra, G., Alarcón, E.P., López, G., Ramírez, D.M., Jaramillo, G.E. (2010), Detección de ectoparásitos en aves silvestres evaluadas en Medellìn (Colombia). Rev Colomb Cienc Pecu; 24:29-37

Pinkus, M. (2010). El hombre y los artrópodos: un vínculo inalienable, Península, Vol. 5( 2):81-100. , Retrieved January 18, 2021, from http://www.scielo.org.mx/scielo.ph $\mathrm{p}$ ?script $=$ sci_arttext\&pid $=\mathrm{S} 1870$ 57662010000200004

Rodríguez-Ortega, L.T., EquihuaMartínez, A., Nieto-Aquino, R., Pro-Martínez, A., RodríguezOrtega, A. (2018). Infestación De Piojos (Phthiraptera) En Gallinas, Gallos Y Pollos (Gallus Gallus Domesticus, Linnaeus). Folia Entomológica Mexicana (nueva serie),4(2), 80-84. Retrieved January 18，2021， from http://www.folia.socmexent.org/rev ista/Num\%202018_2/FEM_4(2)_8 0-84_2018.pdf

Saavedra-Orjuela, A., Arévalo-Barreto, S., Soler-Tovar, D. (2014). Ectoparásitos del orden Phthiraptera en aves silvestres. MEM. CONF. INTERNA MED. APROVECH. FAUNA SILV. EXÓT. CONV. • 10: 2. 5-27.

Santa Cruz, A., Agüero, M.C.,
González, J.A., Comolli, J.A., Cayo, D., Roux, J.P. (2008). Descripción de la morfología externa por microscopía de luz y electrónica de barrido de Megninia ginglymura, Megnin, 1877 en faisán de collar (Phasianus torquatus), en un criadero de Corrientes, Argentina. REDVET. Revista Electrónica de Veterinaria, 9(9), 1-9. Retrieved January 18, 2021, from https://www.redalyc.org/pdf/636/63 617329002.pdf

Sparagano, O., Waap, H., Lima, J.F. (2016). El impacto del ácaro rojo en la producción de huevos. Albéitar: publicación veterinaria independiente, (199), 26-27. Retrieved January 18, 2021, from https://bmeditores.mx/avicultura/elimpacto-del-acaro-rojo-en-laproduccion-de-

huevos/\#: :text=EL\%20IMPACTO $\% 20$ EN\%20LA \%20PRODUCCI\%

C3\%93N,las\%20infestaciones\%20s on $\% 20$ muy\%20altas).

Yates, D.M., Wolstenholme A.J. (2004). An ivermectin-sensitive glutamate-gated chloride channel subunit from Dirofilaria immitis. Int J Parasitol. 34(9):1075-81.

Zucca, P., Delogu, M. (2010). Enfermedades infecciosas y parasitarias. pp. 309-392. En: Samour, J. Medicina aviaria 2da edición. Elsevier. España. ISBN 8480866411. 